\title{
Comparison of Electronic Transport Characterization Methods for Alkanethiol Self-Assembled Monolayers ${ }^{\dagger}$
}

\author{
Takhee Lee,*,: Wenyong Wang, James F. Klemic, Jingshen J. Zhang, Jie Su, and \\ Mark A. Reed* \\ Departments of Electrical Engineering, Applied Physics, and Physics, Yale University, P.O. Box 208284, \\ New Haven, Connecticut 06520 \\ Received: February 2, 2004; In Final Form: April 2, 2004
}

\begin{abstract}
Room-temperature charge transport is investigated for various-length alkanethiol self-assembled monolayers using three different characterization methods, in which lateral areas span from the nanometer to the micrometer scale. In each method, the measured current-voltage characteristics are analyzed with metal-insulatormetal tunneling models. Transport parameters are determined where possible and compared across methods, as well as to previously reported values. Advantages and limitations of each method for characterizing molecular junctions are highlighted.
\end{abstract}

\section{Introduction}

There has been recent interest in molecular-scale charge transport, and the physics underlying molecular-scale charge transport remains an exciting and open area of active research. ${ }^{1-3}$ For example, when a molecular layer with a large HOMOLUMO gap (HOMO, highest occupied molecular orbital; LUMO, lowest unoccupied molecular orbital) is sandwiched between two metal contacts, a well-defined metal-insulatormetal $(\mathrm{M}-\mathrm{I}-\mathrm{M})$ tunneling is expected. One molecular system that has been studied extensively is the alkanethiol $\left[\mathrm{CH}_{3}-\right.$ $\left.\left(\mathrm{CH}_{2}\right)_{n-1} \mathrm{SH}\right]$ self-assembled monolayer (SAM). ${ }^{4}$ Scanning tunneling microscopy, 5,6 conducting atomic force microscopy (CAFM) ${ }^{7-10}$ mercury-drop junctions, ${ }^{11-14}$ cross-wire junctions, ${ }^{15}$ and electrochemical methods ${ }^{16-18}$ have been used to investigate electron transport through alkanethiol SAMs. The charge transport through alkanethiol SAMs is expected to be tunneling because the Fermi levels of the contacts lie within the large HOMO-LUMO gap $(\sim 8 \mathrm{eV})$ of these short $(1-2.5$ $\mathrm{nm}$ ) alkanethiol molecules. ${ }^{19-21}$ It has been recently shown that tunneling is the main conduction mechanism through alkanethiol SAMs in the absence of parasitic parallel paths. ${ }^{22}$

Unfortunately, the characterization of charge transport in molecular-scale electronic systems has to date been dependent on specifics of device fabrication and preparation, as evidenced by the wide dispersion of results for the alkanethiol molecular system, ${ }^{5-14,16-18}$ summarized in Table 1 . None of these studies have attempted to resolve the obvious differences between the methods.

In this study, tunneling characteristics of molecular $\mathrm{M}-\mathrm{I}-\mathrm{M}$ systems are investigated using three different methods: (1) conducting atomic force microscopy of a SAM on a planar surface (defined as "CAFM-planar"), (2) a system using an optically defined etched mesa structure of micrometer lateral dimensions ("monolithic mesa"), and (3) a nanometer-sized system that has successfully been used to characterize a variety

\footnotetext{
† Part of the special issue "Alvin L. Kwiram Festschrift"

* To whom correspondence may be addressed. E-mail: mark.reed@ yale.edu (M.A.R.); takhee.lee@yale.edu (T.L.).

†resent address: Department of Materials Science and Engineering, Gwangju Institute of Science and Technology, 1 Oryong-dong, Buk-gu, Gwangju, 500-712, Korea. E-mail: tlee@gist.ac.kr.
}

of molecules, a nanopore. ${ }^{22,27,28}$ In each method, alkanethiol SAMs were formed on $\mathrm{Au}$ electrode surfaces in an identical manner via chemisorbed $\mathrm{Au}$-thiol bond formation under similar chemical conditions (the other electrode contact was made via physisorption), creating a $\mathrm{M}-\mathrm{I}-\mathrm{M}$ system. Each method was then characterized using methods unique to the geometry and scale of the system. The measured $I(V)$ data are compared with theoretical models of $\mathrm{M}-\mathrm{I}-\mathrm{M}$ tunneling. $I(V)$ measurements on various alkanethiols of different molecular lengths are also performed for the study of length-dependent tunneling behavior. Tunneling current densities, tunneling barrier height, electron effective mass, and decay coefficients are determined where possible from measured results and compared between the different structures. A comparison of results and subsequent analysis from each method is presented, and advantages and limitations are discussed.

\section{Experimental Section}

2.1. Alkanethiol Deposition. For our experiments, a $\sim 5 \mathrm{mM}$ alkanethiol solution was prepared by adding $\sim 10 \mu \mathrm{L}$ alkanethiols into $\sim 10 \mathrm{~mL}$ ethanol. ${ }^{29}$ The deposition was done in solution for 1-2 days inside a nitrogen-filled glovebox with an oxygen level of less than $100 \mathrm{ppm}$. Alkanethiol molecules of varying molecular lengths, octanethiol $\left(\mathrm{CH}_{3}\left(\mathrm{CH}_{2}\right)_{7} \mathrm{SH}\right.$, denoted as $\mathrm{C}$, for the number of alkyl units), decanethiol $\left(\mathrm{CH}_{3}\left(\mathrm{CH}_{2}\right)_{9} \mathrm{SH}, \mathrm{C} 10\right)$, dodecanethiol $\left(\mathrm{CH}_{3}\left(\mathrm{CH}_{2}\right)_{11} \mathrm{SH}, \mathrm{C} 12\right)$, tetradecanethiol $\left(\mathrm{CH}_{3}\left(\mathrm{CH}_{2}\right)_{13} \mathrm{SH}, \mathrm{C} 14\right)$, and hexadecanethiol $\left(\mathrm{CH}_{3}\left(\mathrm{CH}_{2}\right)_{15} \mathrm{SH}, \mathrm{C} 16\right)$ were used to form the active molecular component. ${ }^{29}$ As a representative example, the chemical structure of octanethiol is shown in Figure 1c.

2.2. Fabrication and Characterization. Charge-transport measurements on alkanethiol SAMs were performed using three types of methods, as schematically illustrated in Figure 1: (1) The CAFM-planar method (Figure 1a) presents an atomically flat $\mathrm{Au}$ surface $(\mathrm{Au}(250 \mathrm{~nm}) / \mathrm{Cr}(3 \mathrm{~nm})$ thickness $)$ on glass prepared by hydrogen flame annealing. (2) The monolithic mesa (Figure $1 \mathrm{~b}$ ) is a $3.3 \times 3.3 \mathrm{~mm}^{2}$ silicon chip with microscale structures that was fabricated by conventional microlithography. Figure 2 shows a series of images of microfabricated monolithic mesas. The size of the electrode surface available for SAM formation varies from $1 \mu \mathrm{m}$ to $100 \mu \mathrm{m}$. In these devices, the 
TABLE 1: Summary of Alkanethiol Tunneling Characteristic Parameters ${ }^{a}$

\begin{tabular}{|c|c|c|c|c|c|}
\hline junction & $\beta\left(\AA^{-1}\right)$ & $J\left(\mathrm{~A} / \mathrm{cm}^{2}\right)$ at $1 \mathrm{~V}$ & $\Phi_{\mathrm{B}}(\mathrm{eV})$ & technique & ref \\
\hline (bilayer) monothiol & $0.87 \pm 0.1$ & $25-200^{b}$ & $2.1^{f}$ & Hg-junction & 11 \\
\hline (bilayer) monothiol & $0.71 \pm 0.08$ & $0.7-3.5^{b}$ & & $\mathrm{Hg}$-junction & 13 \\
\hline monothiol & $0.79 \pm 0.01$ & $1500 \pm 200^{c}$ & $1.4^{f}$ & solid $M-I-M$ & 22 \\
\hline monothiol & 1.2 & & & STM & 5 \\
\hline dithiol & $0.8 \pm 0.08$ & $3.7-5 \times 10^{5 d}$ & $5 \pm 2^{g}$ & STM & 6 \\
\hline monothiol & $0.73-0.95$ & $1100-1900^{e}$ & $2.2^{f}$ & CAFM & 7 \\
\hline monothiol & $0.64-0.8$ & $10-50^{e}$ & $2.3^{f}$ & CAFM & 9 \\
\hline dithiol & $0.46 \pm 0.02$ & $3-6 \times 10^{5 d}$ & $1.3-1.5^{f}$ & CAFM & 10 \\
\hline monothiol & $1.37 \pm 0.03$ & & $1.8^{g}$ & tuning fork AFM & 23 \\
\hline monothiol & $0.97 \pm 0.04$ & & & electrochemical & 16 \\
\hline monothiol & 0.85 & & & electrochemical & 17 \\
\hline monothiol & $0.91 \pm 0.08$ & & & electrochemical & 18 \\
\hline monothiol & 0.76 & $2 \times 10^{4}(\text { at } 0.1 \mathrm{~V})^{d}$ & $1.3-3.4^{h}$ & theory & 24 \\
\hline monothiol & 0.76 & & & theory & 25 \\
\hline monothiol & 0.79 & & & theory & 26 \\
\hline
\end{tabular}

${ }^{a}$ Note: Some decay coefficients $\beta$ were converted into the unit of $\AA^{-1}$ from the unit of per methylene. Current densities $(J)$ for C12 monothiol or dithiol at $1 \mathrm{~V}$ are extrapolated from published results for other length molecules by using the conductance $\propto \exp (-\beta d)$ relationship. Although current density may be crudely converted to current per molecule by multiplying current density with the area of single molecule $\left(0.217 \mathrm{~nm}^{2}\right)$, this assumes the current flowing through many molecules is directly proportional to the number of molecules. This assumption requires a more thorough quantitative analysis as other mechanisms may affect charge transport in many-molecule systems. ${ }^{47}{ }^{b}$ Junction areas estimated by optical microscope. ${ }^{c}$ Junction areas estimated by SEM. ${ }^{d}$ Junction areas estimated by assuming single molecule. ${ }^{e}$ Junction areas estimated by Hertzian contact theory. ${ }^{f}$ Barrier height $\Phi_{\mathrm{B}}$ values were obtained from the Simmons equation. ${ }^{g}$ Barrier height $\Phi_{\mathrm{B}}$ values were obtained from bias-dependence of $\beta$. ${ }^{h}$ Barrier height $\Phi_{\mathrm{B}}$ values were obtained from a theoretical calculation.

(a)

(b)
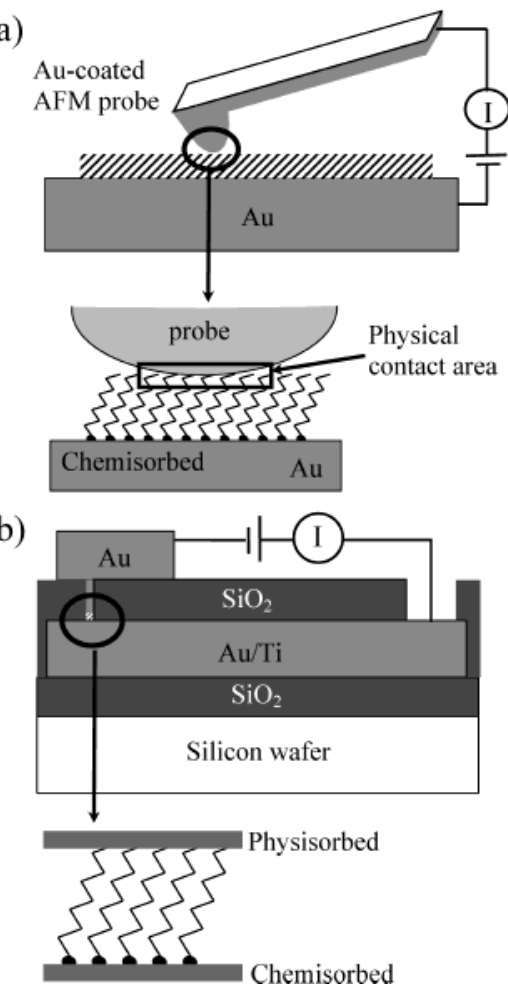

(c)
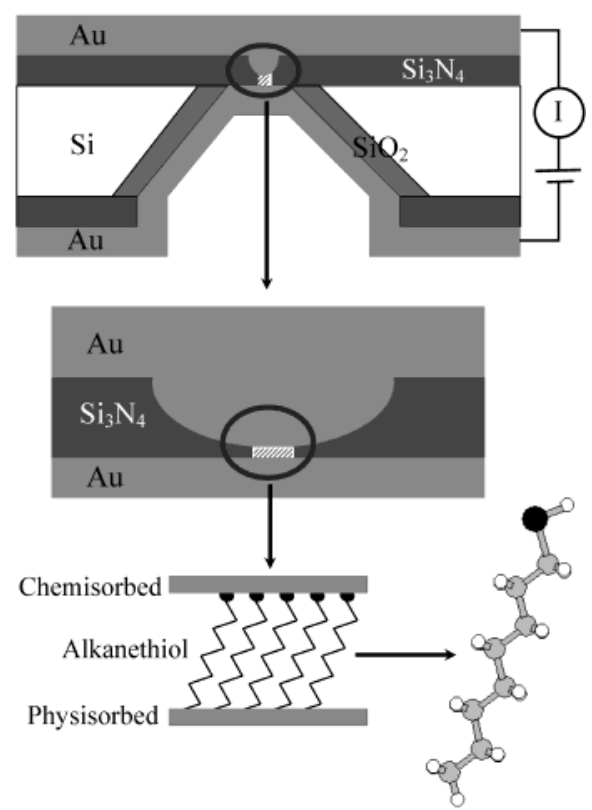

Figure 1. Schematics of three types of characterization methods used in this study. (a) CAFM-planar: conducting atomic force microscopy on alkanethiol SAMs on an atomically flat Au surface. (b) Monolithic mesa: the typical junction area is $2960 \mathrm{~nm}$ in diameter. (c) Nanopore: top schematic is the cross section of a silicon wafer with a nanometer-scale pore etched through a suspended silicon nitride membrane. Middle and bottom schematics show a Au/SAM/Au junction (area $\sim 45 \mathrm{~nm}$ in diameter) formed in the pore area. The structure of octanethiol is shown as an example.

$\mathrm{Au}$ surface is exposed by etching through a $100 \mathrm{~nm}$ thick $\mathrm{SiO}_{2}$ overlayer using standard microlithographic fabrication techniques. The diameter of these smallest circular Au structures was determined as $960 \pm 60 \mathrm{~nm}$ from AFM images on various devices. (3) The nanopore (Figure 1c) is a single nanoscale device structure on a $3.5 \times 3.5 \mathrm{~mm}^{2}$ silicon chip and has been described in detail previously. ${ }^{22,27,28,30}$ The average diameter of device size (junction area) was determined to be $45-50 \mathrm{~nm}$ from scanning electron microscope (SEM) images. ${ }^{22}$
For the CAFM-planar method, alkanethiol SAMs were deposited and then the surface was characterized via the CAFM technique at room temperature in an ambient environment. ${ }^{31,32}$ For nanopore and monolithic mesa methods, alkanethiol SAMs were formed (chemisorbed) on exposed Au surfaces, and the other (physisorbed) contact $(\sim 100 \mathrm{~nm} \mathrm{Au})$ was made by thermal evaporation under the pressure of $\sim 10^{-8}$ Torr. The evaporation was done with the chips in contact with a liquid nitrogen cooled cold stage in order to avoid thermal damage to the molecular 
(a)

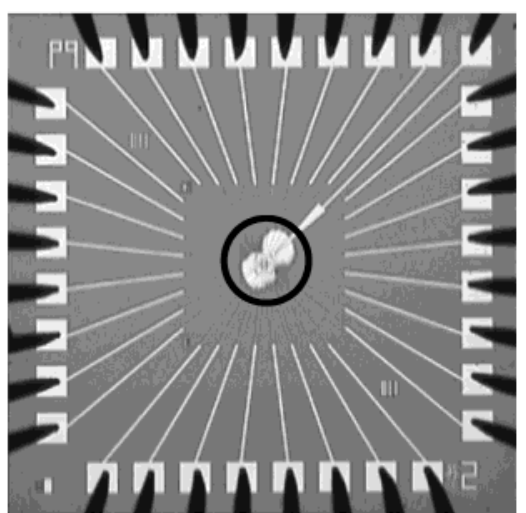

(c)

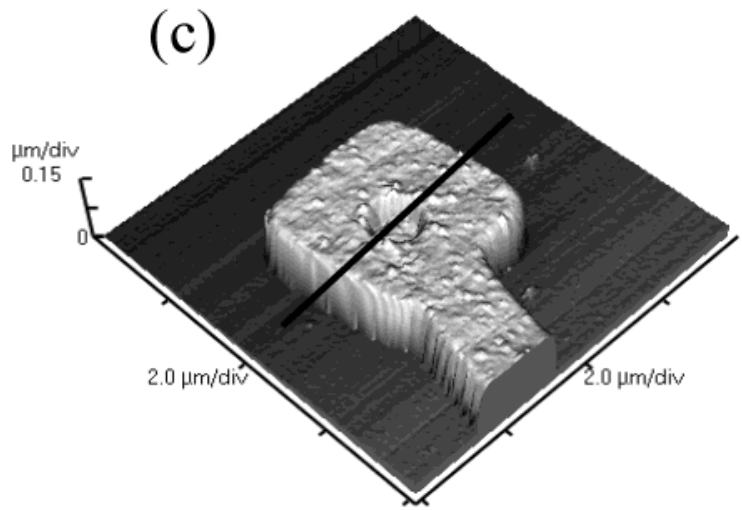

(b)

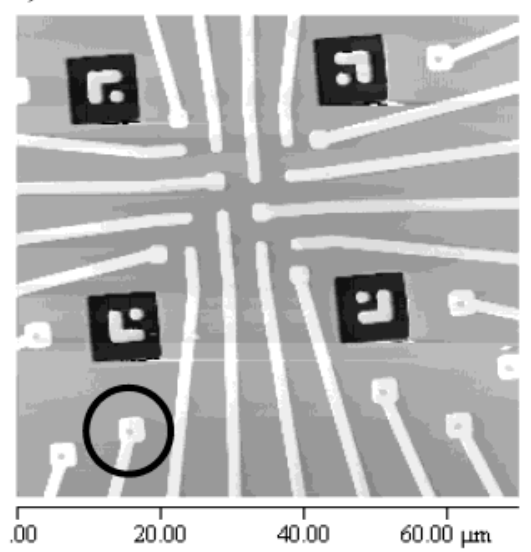

(d)

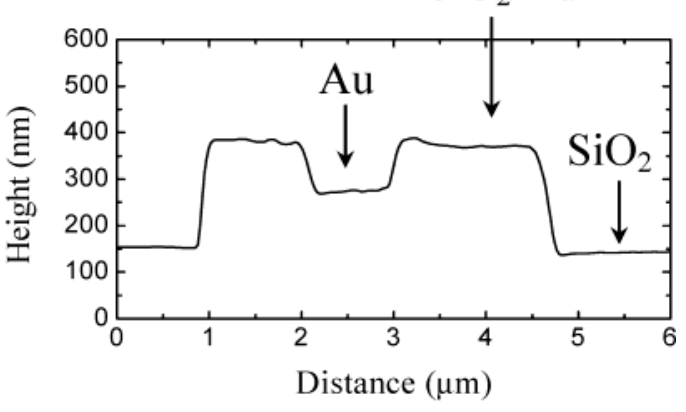

Figure 2. Monolithic mesa devices. (a) Optical image of a $3.3 \mathrm{~mm} \times 3.3 \mathrm{~mm}$ chip after top Au metallization (circled in the center). Black objects around the chip are probing needles. (b) AFM image of central area of a chip (circled in a before the top Au metallization). (c) $8 \mu \mathrm{m} \times 8 \mu \mathrm{m}$ AFM image showing a microscale device (circled in b). (d) A cross-sectional profile obtained along the line shown in c.

layer. $^{22,33}$ Room-temperature two-terminal DC $I(V)$ measurements were performed using semiconductor parameter analyzers (HP4145B and Agilent 4156B).

\section{Theoretical Basis}

3.1. Tunneling Model. The simplest model to describe the tunneling behavior through alkanethiol $\mathrm{M}-\mathrm{I}-\mathrm{M}$ systems is the Simmons model. ${ }^{34,35}$ The temperature-independent tunneling current density $J$ through an alkanethiol tunnel barrier is expressed as ${ }^{11,22,34}$

$$
\begin{array}{r}
J= \\
\left(\frac{e}{4 \pi^{2} \hbar d^{2}}\right)\left\{\left(\Phi_{\mathrm{B}}-\frac{e V}{2}\right) \exp \left[-\frac{2(2 m)^{1 / 2}}{\hbar} \alpha\left(\Phi_{\mathrm{B}}-\frac{e V}{2}\right)^{1 / 2} d\right]-\right. \\
\left.\left(\Phi_{\mathrm{B}}+\frac{e V}{2}\right) \exp \left[-\frac{2(2 m)^{1 / 2}}{\hbar} \alpha\left(\Phi_{\mathrm{B}}+\frac{e V}{2}\right)^{1 / 2} d\right]\right\}
\end{array}
$$

where $m$ is electron mass, $d$ is barrier width, $\Phi_{\mathrm{B}}$ is barrier height, $V$ is the applied bias, and $h(2 \pi \hbar)$ is Planck's constant. For molecular M-I-M systems, the Simmons model has been modified with a parameter $\alpha .^{11,22}$ The $\alpha$ parameter provides either a way of applying the tunneling model of a rectangular barrier to tunneling through a nonrectangular barrier ${ }^{11}$ or an adjustment to account for the effective mass $\left(\mathrm{m}^{*}\right)$ of the tunneling electrons through a rectangular barrier., ${ }^{9,22,26,36}$ By fitting individual $I(V)$ data using eq $1, \Phi_{\mathrm{B}}$ and $\alpha$ values can be obtained.
In the low-bias region, eq 1 can be approximated as 22,34

$$
J \approx\left(\frac{\left(2 m \Phi_{\mathrm{B}}\right)^{1 / 2} e^{2} \alpha}{h^{2} d}\right) V \exp \left[-\frac{2(2 m)^{1 / 2}}{\hbar} \alpha\left(\Phi_{\mathrm{B}}\right)^{1 / 2} d\right]
$$

where the tunneling decay coefficient $\beta$ can be defined from $J$ $\propto(1 / d) \exp (-\beta d)$ as

$$
\beta=\frac{2(2 m)^{1 / 2}}{\hbar} \alpha\left(\Phi_{\mathrm{B}}\right)^{1 / 2}
$$

Thus $\beta$ values can be calculated using $\Phi_{\mathrm{B}}$ and $\alpha$ values obtained from $I(V)$ data fittings.

3.2. Length-Dependent Tunneling. The conductance $G$ through alkanethiol SAMs has shown an exponential dependence on the barrier width $d$ as ${ }^{5-14,22}$

$$
G \propto \exp (-\beta d)
$$

where $\beta$ is the decay coefficient. This equation has been generally used to determine $\beta$ values from length-dependent measurements and analysis. ${ }^{5-14,22}$

One should note that $\beta$ values obtained via eq $2 \mathrm{~b}$ are from fitting individual $I(V)$ measurements for specific molecules, while $\beta$ values from eq 3 are obtained from fitting conductance data collected from various molecules with different lengths.

\section{Results}

4.1. CAFM-Planar Method. $I(V)$ characterization was performed on alkanethiol SAMs formed on planar annealed gold 


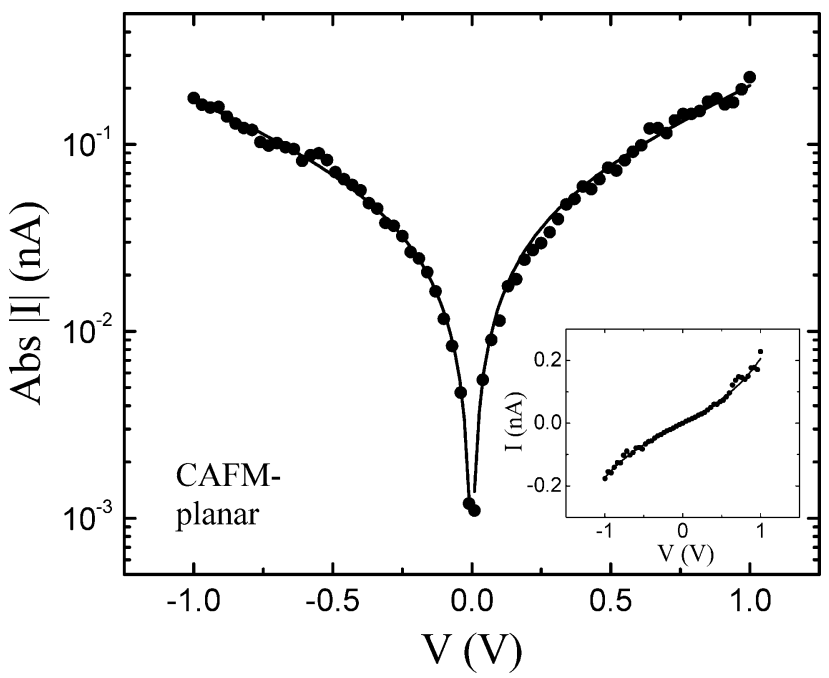

Figure 3. $I(V)$ characteristics of the $\mathrm{C} 12 \mathrm{SAM}$ formed in the CAFMplanar structure at probe-loading force of $10 \mathrm{nN}$ in semilog scale and linear scale (inset)

substrates by the CAFM technique, as illustrated in Figure 1a. This CAFM-planar method presents an atomically flat uniform surface for optimal probing of SAM-deposited samples using AFM techniques. The probe is in direct contact with the sample, which a priori would appear to minimize vacuum tunneling effects and ensure that the applied bias represents the voltage drop directly across the coated substrate (unlike STM measurements which have an unknown impedance tunneling gap).

Figure 3 shows $I(V)$ characteristics measured (symbols) on $\mathrm{C} 12$ with a loading force of $10 \mathrm{nN}$ at room temperature. A curve fitting using the tunneling model (eq 1) is plotted as a solid curve in this figure, demonstrating that the mechanism appears to be tunneling. For this device, the optimum fitting parameters were found as $\Phi_{\mathrm{B}}=2.57 \mathrm{eV}$ and $\alpha=0.56$ for positive bias region and $\Phi_{\mathrm{B}}=2.93 \mathrm{eV}$ and $\alpha=0.53$ for negative bias region. The $I(V)$ plots exhibit a slight asymmetry, which is consistent with the $\mathrm{M}-\mathrm{I}-\mathrm{M}$ system under test (one end of the molecule is chemisorbed to the bottom contact and the other end is in physical contact to the top probe). The conductance (in the lowbias range, $V \leq|0.5| V$ ) is $7.4 \mathrm{nS}$, and the current density (at $1.0 \mathrm{~V}$ ) is $\sim 110 \mathrm{~A} / \mathrm{cm}^{2}$, assuming a (calculated) contact junction diameter of $\sim 16.6 \mathrm{~nm}$ using methods described shortly. This current density is in reasonable agreement with those listed in Table 1.

To verify a tunneling dependence, the $I(V)$ characteristics can be further examined by the length-dependent tunneling behavior (eq 3). Figure 4 is a semilog plot of the low-bias regime conductance as a function of the molecular length for C8, C10, $\mathrm{C} 12, \mathrm{C} 14$, and C16 alkanethiols at a fixed force of $20 \mathrm{nN}$. The error ranges were determined statistically from different measurements on various sample positions. The molecular lengths used in this plot are 13.3, 15.7, 18.2, 20.7, and 23.2 $\AA$ for from C8 to C16 alkanethiols (each molecular length was determined by adding an $\mathrm{Au}$-thiol bonding length to the length of molecule). ${ }^{8}$ Note that these lengths assume "through-bond" tunneling, that is, along the tilted molecular chains between the metal contacts. ${ }^{8,9,18,37}$ The conductance shows an overall exponential dependence on molecular length corresponding to a $\beta$ value of $1.04 \pm 0.20 \AA^{-1}$. This value is in reasonable agreement with previously reported $\beta$ values listed in Table 1 .

From these results, including the good fit to the Simmons model, it may appear that the CAFM method is a valid, appealing (due to imaging capability), and easily accessible

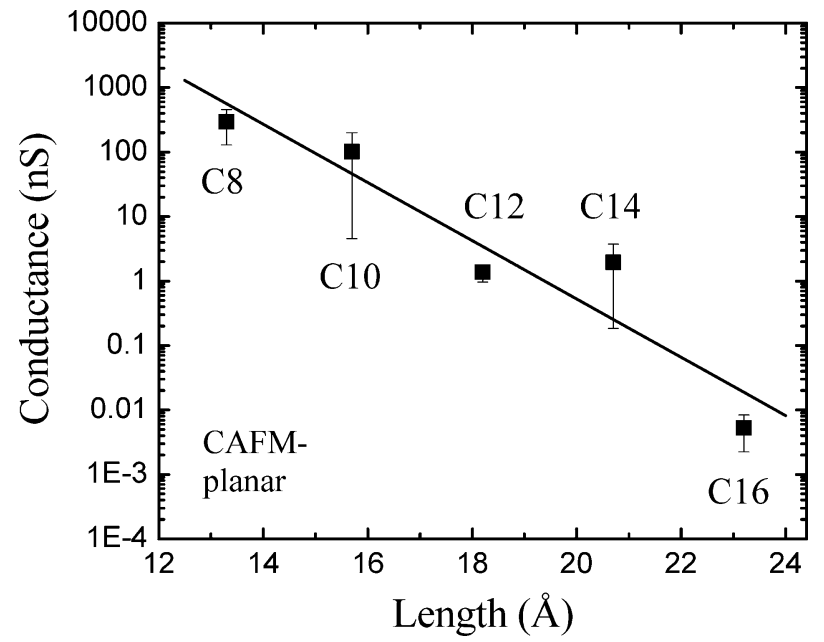

Figure 4. Semilog plot of conductance of C8, C10, C12, C14, and C16 SAMs formed in the CAFM-planar structure vs molecular lengths (at fixed $F=20 \mathrm{nN}$ ). The line through the data point is exponential fitting.

approach to measuring electronic transport across monolayers, however, results require careful analysis. Consider the $I(V)$ data for $\mathrm{C} 12$ measured with increasing probe-loading forces in Figure 5a. The current increases with increasing force. Although this is qualitatively expected due to increasing contact junction area for increasing force, the current density values should remain constant if probe does not penetrate or deform the molecular layer. This assumption must be more carefully analyzed quantitatively.

The current density may be calculated by estimating a contact junction area for a given loading force using a Hertzian elastic contact model. ${ }^{7,9,38,39}$ The radius $a$ of the contact junction area by the CAFM probe may be estimated (when adhesion between the probe and sample is considered) as ${ }^{39}$

$$
\begin{aligned}
& a^{3}=(R / K) P_{\mathrm{n}}= \\
& (R / K)\left\{P+3 \Gamma \pi R+\left(6 \Gamma \pi R P+(3 \Gamma \pi R)^{2}\right)^{1 / 2}\right\}
\end{aligned}
$$

where $R$ is the radius of the CAFM probe tip (for our system, $90 \pm 20 \mathrm{~nm}$ from SEM images on various probes), $P_{\mathrm{n}}$ is the net force (sum of applied loading force $\mathrm{P}$ and terms due to adhesion force), $\Gamma=2 P_{\mathrm{c}} / 3 \pi R$ is the adhesion energy per unit area related to adhesion force $P_{\mathrm{c}}\left(P_{\mathrm{c}}=16 \mathrm{nN}\right.$ observed from force-distance characterization), and $K=\left({ }^{4} / 3\right)\left[\left(1-v_{1}^{2}\right) / E_{1}+\right.$ $\left.\left(1-v_{2}^{2}\right) / E_{2}\right]^{-1}\left(E_{1}, v_{1}, E_{2}\right.$, and $v_{2}$ are Young's modulus and Poisson's ratio of the sample and Au-coated AFM probe, respectively). Appropriate $E_{1}, v_{1}, E_{2}$, and $v_{2}$ values are not available, but assuming $E_{1} \approx 10 \mathrm{GPa},{ }^{39 a, 40 \mathrm{a}} E_{2} \approx 69 \mathrm{GPa},{ }^{40 \mathrm{~b}}$ and $v_{1} \approx v_{2} \approx 0.33^{9,40 \mathrm{c}}$ compared to similar materials and structures, K may be calculated as $\sim 13 \mathrm{GPa}$. Note others have either not considered or simplified the adhesion terms between probe and sample, obtaining the radius of contact junction area as simply $(R P / K)^{1 / 3} \cdot{ }^{7,9}$ An electrostatic capacitive force between sample and probe has been reported to cause an additional adhesive force ${ }^{41}$ however, it does not significantly contribute to a modification in the contact junction area due to the relatively low applied bias range employed in this study.

By use of eq 4 and $I(V)$ data in Figure 5a, the diameters of the contact junction areas were estimated to be $\sim 16.0,16.6$, 17.1, and $17.7 \mathrm{~nm}$, with current densities for $\mathrm{C} 12$ at $1.0 \mathrm{~V}$ of $\sim 40,110,200$, and $510 \mathrm{~A} / \mathrm{cm}^{2}$ for applied forces of 5, 10, 15, and $20 \mathrm{nN}$, respectively. Note that the current densities are not 

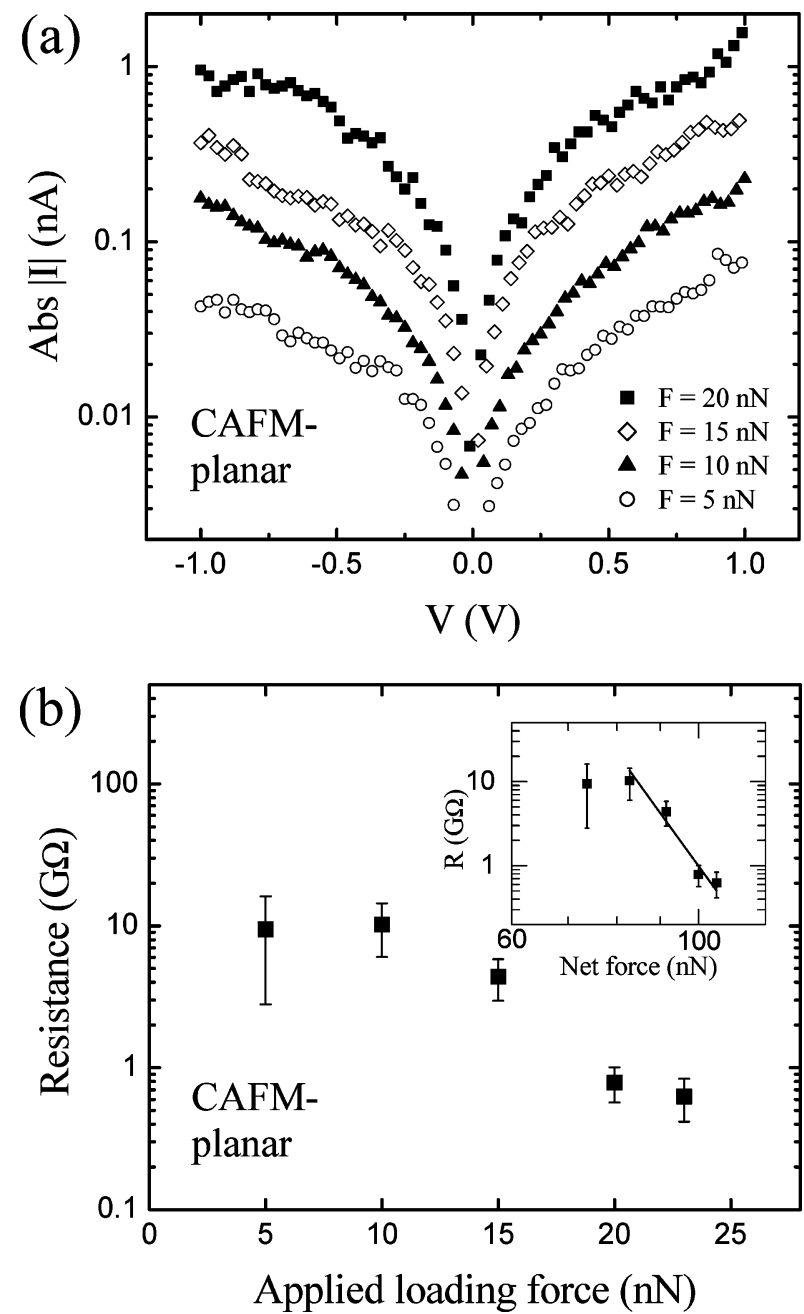

Figure 5. (a) $I(V)$ characteristics of the $\mathrm{C} 12 \mathrm{SAMs}$ formed in the CAFM-planar structure for probe-loading forces from 5 to $20 \mathrm{nN}$. (b) Semilog plot of resistance of C12 SAMs vs loading force. Inset shows a plot of the $\log ($ resistance, $R)$ vs $\log \left(\right.$ net force, $\left.F^{*}\right)$ with a line fit.

constant but increase with increasing force, which implies a partial penetration or deformation of the SAM layer by the probe tip. $^{42}$

This can be checked with a resistance-force relationship. According to eq 4 , the resistance should be proportional to $P_{\mathrm{n}}{ }^{-2 / 3}$ if there is no SAM penetration or deformation. ${ }^{7}$ The resistance as a function of the net force is plotted in semilog scale in Figure 6a. A plot of $\log (R)$ vs $\log \left(P_{\mathrm{n}}\right)$, inset in Figure $6 \mathrm{~b}$, gives an exponent of $\sim-14$, instead of $-2 / 3$, which is expected for the case of no penetration or deformation of the molecular layer. ${ }^{43}$ This indicates that the CAFM tip in this study not only creates a force-dependent contact junction area ${ }^{41}$ but also complicates the analysis by penetration and/or deformation of the molecular layer. ${ }^{42}$

It has been reported that deformed SAMs give through-bond tunneling characteristics identical to nondeformed SAMs. ${ }^{9}$ However, there is no independent experimental evidence that deformation caused by a CAFM tip only changes tilt angle, without structural change or damage of a SAM. A detailed fit of the $I(V)$ characteristics (Figure 3) gave $\left\{\Phi_{\mathrm{B}}=2.57 \mathrm{eV}, \alpha=\right.$ $0.56\}$ and $\left\{\Phi_{\mathrm{B}}=2.93 \mathrm{eV}, \alpha=0.53\right\}$ for positive and negative bias regimes, respectively, which differ greatly from the values of $\left\{\Phi_{\mathrm{B}}=1.39 \pm 0.01 \mathrm{eV}, \alpha=0.65 \pm 0.01\right\}$ obtained from temperature-variable $I(V)(I(V, T))$ and length-dependent measurements using the nanopore method. ${ }^{22}$ By use of these published values, one then obtains a contact area diameter of

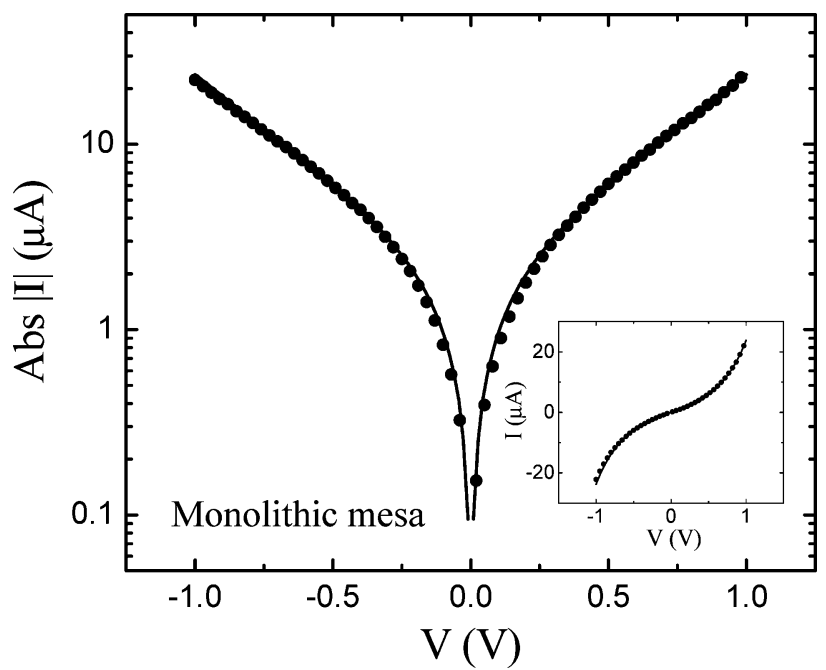

Figure 6. $I(V)$ characteristic of the $\mathrm{C} 12 \mathrm{SAM}$ formed in the monolithic mesa structure in semilog scale and linear scale (inset).

$\sim 4.1 \mathrm{~nm}$ (using eq 1), significantly different from the diameter $(\sim 16.6 \mathrm{~nm})$ obtained with the widely used estimation procedure for contact junction area (eq 4). These inconsistencies indicate a problem with this CAFM method, suggesting that the transport we have characterized through a deformed SAM involves more than just through-bond tunneling.

A thorough study to determine transport parameters requires not only verification in the nondeformation regime but, more importantly, variable temperature studies to eliminate parasitic leakage or other transport mechanisms (which applies to both the deformation or nondeformation regimes). Because of the relative experimental difficulty in achieving this (to date, this has not been reported by others), it eliminates the apparent advantages of the CAFM technique. Without doing both adhesion force analysis to rule out deformation or penetration and a complimentary (and simultaneous) temperature dependent characterization, transport results from CAFM measurements should be considered carefully and treated as not broadly applicable to determining molecular conductivity properties. Thus, agreement of derived values (such as $\beta$ ) with other published results must be viewed as fortuitous in part due to the relative large uncertainty in the derived values.

4.2. Monolithic Mesa Method. $I(V)$ characterization was also performed on alkanethiol SAMs formed on exposed Au surfaces made by the monolithic mesa method, as illustrated in Figures $1 \mathrm{~b}$ and 2. Each chip fabricated in this method contains multiple exposed electrode surfaces of various sizes that fan out to contact pads comparable for standard IC chip characterization and packaging (e.g., automated probing and wire bonding), hence allowing large numbers of chips and devices to be readily manufactured.

Figure 6 shows a representative $I(V)$ characteristic of a $\mathrm{C} 12$ SAM measured (symbols) with this method. Positive bias corresponds to electrons flowing from the physisorbed $\mathrm{Au}$ contact (top contact in Figure 1b) into the molecules. Similarly, the fitted calculation results using eq 1 are plotted as a solid curve in Figure 6. The optimum fitting parameters were found to be $\left\{\Phi_{\mathrm{B}}=1.32 \pm 0.02 \mathrm{eV}\right.$ and $\left.\alpha=0.64 \pm 0.01\right\}$ where the error ranges of $\Phi_{\mathrm{B}}$ and $\alpha$ are determined by the junction area fluctuations $(960 \pm 60 \mathrm{~nm})$. The current density for this device was estimated using the junction area of $960 \pm 60 \mathrm{~nm}$ in diameter obtained from AFM study; and determined to be 3300 $\pm 400 \mathrm{~A} / \mathrm{cm}^{2}$ (at $1.0 \mathrm{~V}$ ). The $I(V)$ shape and current density appear to follow tunneling transport behavior. 

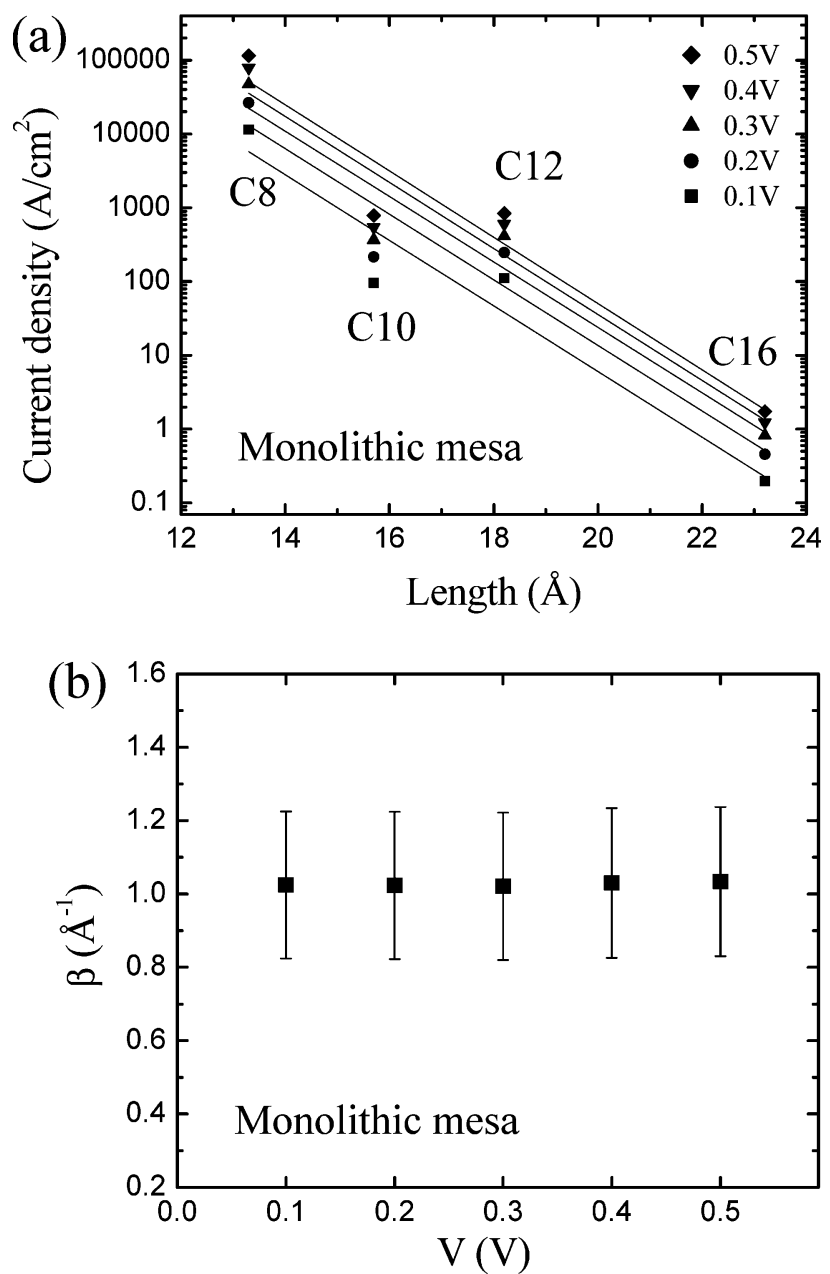

Figure 7. (a) Semilog plot of tunneling current densities (from Figure 6) vs molecular lengths. The lines through the data points are exponential fittings. (b) Plot of $\beta$ vs bias.

Length-dependent tunneling behavior was also investigated. Figure 7a is a semilog plot of tunneling current densities (at various voltages) as a function of the molecular length for various alkanethiols. The tunneling current densities show an overall exponential trend on the molecular length. ${ }^{44}$ The $\beta$ values were determined from the slope at each bias and are plotted in Figure $7 \mathrm{~b}$ where the uncertainties were obtained by linear fitting errors. The $\beta$ values from 0 to $0.5 \mathrm{~V}$ are averaged as $1.02 \pm$ $0.20 \AA^{-1}$. This average $\beta$ value agrees well with the previously reported $\beta$ values listed in Table 1. However, the large uncertainty in $\beta$ values reflects the dispersion in current densities for various alkanethiols and problems with this method.

Specifically, the currents of the C10 and C12 devices were observed to be similar as shown in Figure 8. This is clearly not consistent with a tunneling dependence and may be due to various defects, such as grain boundaries in the SAM (molecular junctions in similar microscale devices have shown large sample-to-sample variations in current densities). ${ }^{45}$ Thus, temperature-dependent measurements were attempted on these devices to further quantify the mechanisms, and it was found that these devices would not thermally cycle (failing within tens of degrees below room temperature), suggesting unstable molecule/metal contacts. Further, a relatively low device yield (typically less than $\sim 0.5 \%$ ) compared to the nanopore method (see below) was observed. This low yield prevents one from exploiting the potential of fabricating large numbers of working devices to be characterized and analyzed using an automated

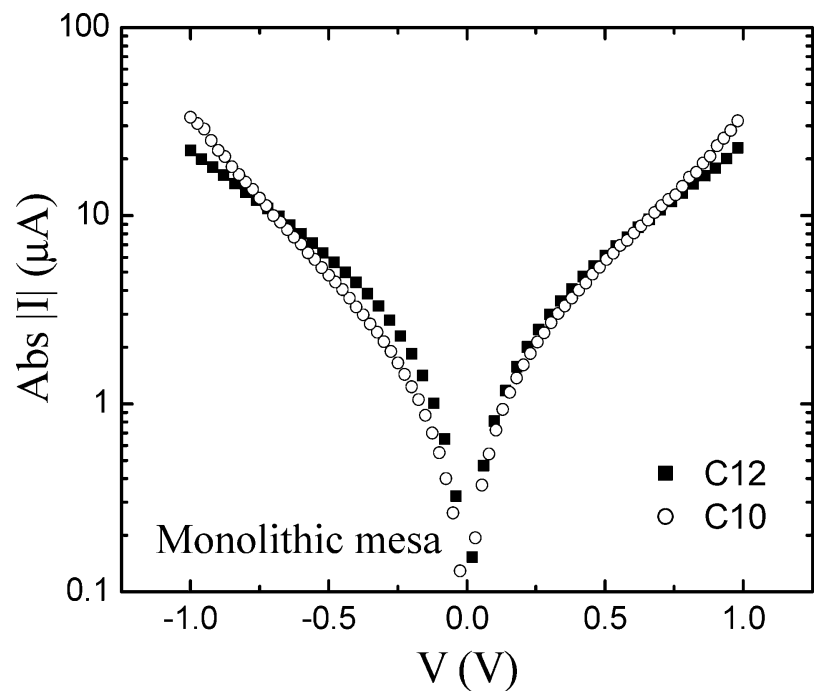

Figure 8. $\mathrm{C} 10$ and $\mathrm{C} 12 \mathrm{I}(\mathrm{V})$ data show similar current values.

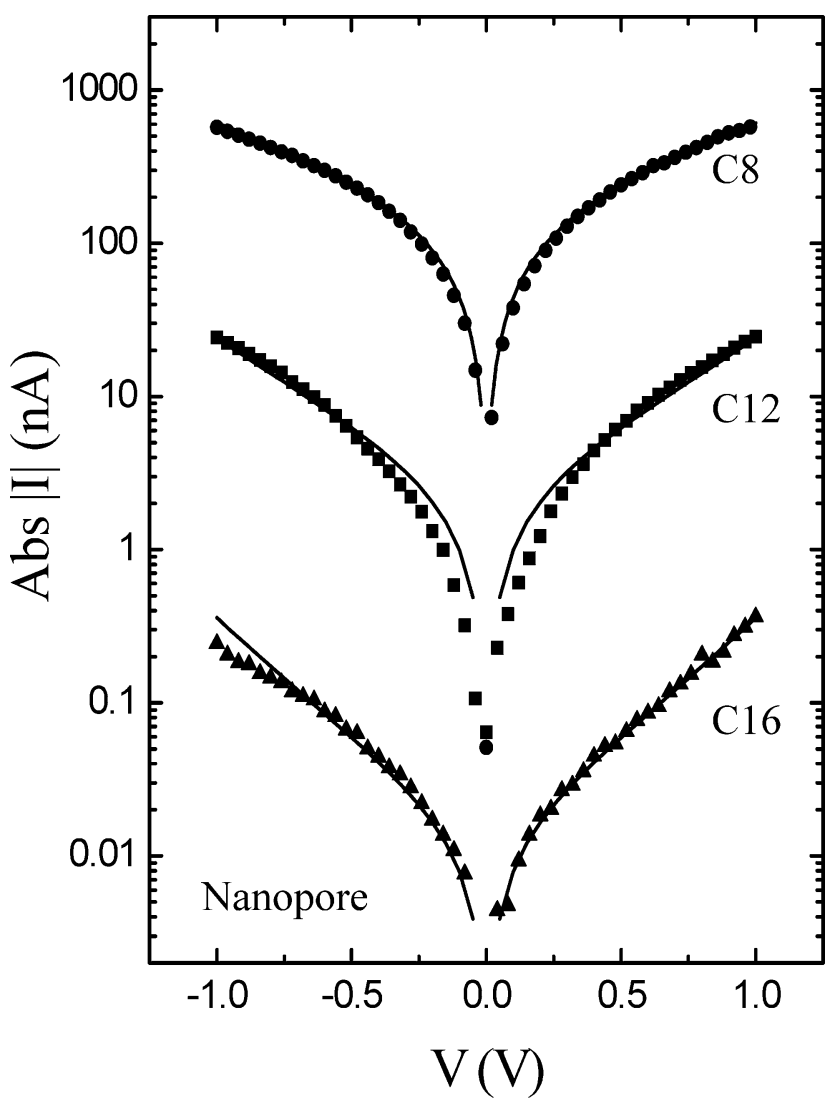

Figure 9. $I(V)$ characteristics of $\mathrm{C} 8, \mathrm{C} 12$, and $\mathrm{C} 16$ SAMs formed in a nanopore structure. Measured data (symbols) are compared with calculations (solid curves) using the optimum fitting parameters of $\Phi_{\mathrm{B}}$ and $\alpha$.

wafer-level IC characterization. Therefore, this monolithic mesa method is not a satisfactory approach for characterizing molecular M-I-M systems.

4.3. Nanopore Method. Figure 9 shows representative $I(V)$ characteristics of $\mathrm{C} 8, \mathrm{C} 12$, and C16 SAMs measured (symbols) with the nanopore method as shown in Figure 1c. Positive bias corresponds to electrons flowing from the physisorbed $\mathrm{Au}$ contact (bottom contact in Figure 1c) into the molecules. By use of the contact area of $50 \pm 8 \mathrm{~nm}$ (for the C8 device) and $45 \pm 2 \mathrm{~nm}$ in diameter (for the $\mathrm{C} 12$ and C16 devices), ${ }^{22}$ the current densities of $31000 \pm 10000,1500 \pm 200$, and $23 \pm$ 

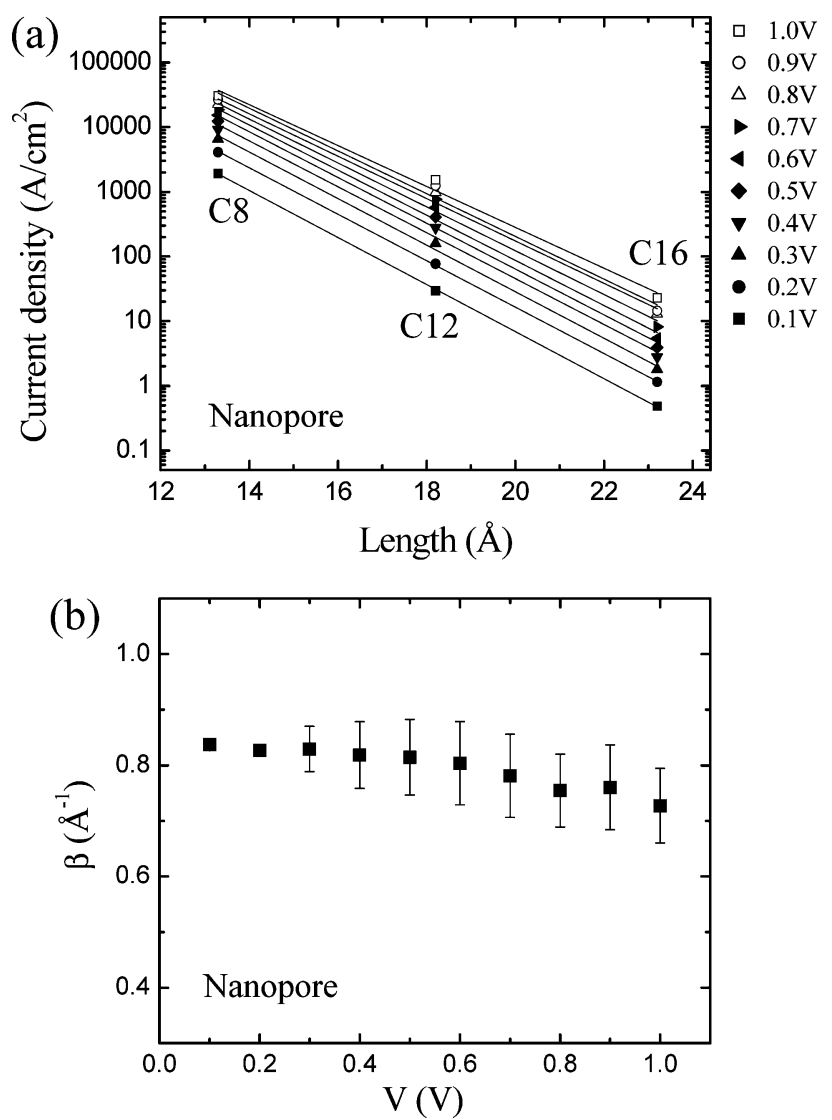

Figure 10. (a) Semilog plot of tunneling current densities (from Figure 7) vs molecular lengths. The lines through the data points are exponential fittings. (b) Plot of $\beta$ vs bias.

$2 \mathrm{~A} / \mathrm{cm}^{2}$ at $1.0 \mathrm{~V}$ are determined for $\mathrm{C} 8, \mathrm{C} 12$, and $\mathrm{C} 16$, respectively. A more ideal (less parasitic) C8 sample supercedes that of previous reports, ${ }^{22}$ and derived parameters from the two data sets agree within a standard error. From previously reported temperature-independent $I(V)$ measurement results using this device structure, tunneling has been shown to be the main conduction mechanism occurring through alkanethiol SAMs. ${ }^{22}$ Similarly, $I(V)$ results were further analyzed using two tunneling models: (1) fitting to the modified Simmons tunneling model (eq 1) and (2) molecular length-dependent tunneling analysis (eq 3). Tunneling parameters derived from these analysis are then compared.

The optimum fitting parameters $\Phi_{\mathrm{B}}$ and $\alpha$ (by minimizing $\left.\chi^{2}\right)$ for the $I(V)$ data from curve fitting with eq 1 were found to be $\left\{\Phi_{\mathrm{B}}=1.83 \pm 0.10 \mathrm{eV}\right.$ and $\left.\alpha=0.61 \pm 0.01\right\},\left\{\Phi_{\mathrm{B}}=1.42\right.$ $\pm 0.04 \mathrm{eV}$ and $\alpha=0.65 \pm 0.01\}$, and $\left\{\Phi_{\mathrm{B}}=1.40 \pm 0.03 \mathrm{eV}\right.$ and $\alpha=0.68 \pm 0.01\}$ for $\mathrm{C} 8, \mathrm{C} 12$, and $\mathrm{C} 16 I(V)$ data, respectively. Uncertainties in these fits are dominated by fluctuations in the sample-to-sample junction area. These calculation results are plotted as solid curves in Figure 9. For the case of a rectangular barrier limit, the $\alpha$ parameter fits presented above correspond to an effective mass $m^{*}\left(=\alpha^{2} m\right)$ of $0.37,0.42$, and $0.46 \mathrm{~m}$ for $\mathrm{C} 8, \mathrm{C} 12$, and $\mathrm{C} 16$, respectively.

Figure 10a is a length-dependent plot; a semilog plot of tunneling current densities at various voltages as a function of the molecular length for these alkanethiols. The tunneling current densities show exponential dependence on molecular length. The $\beta$ values can be determined from the slope at each bias and are plotted in Figure 10b. The uncertainty of an individual $\beta$ value in this plot was obtained from the linear fitting errors. This gives a $\beta$ value from 0.84 to $0.73 \AA^{-1}$ in the bias range from 0.1 to $1.0 \mathrm{~V}$. Increasing the bias voltage effectively lowers the barrier,
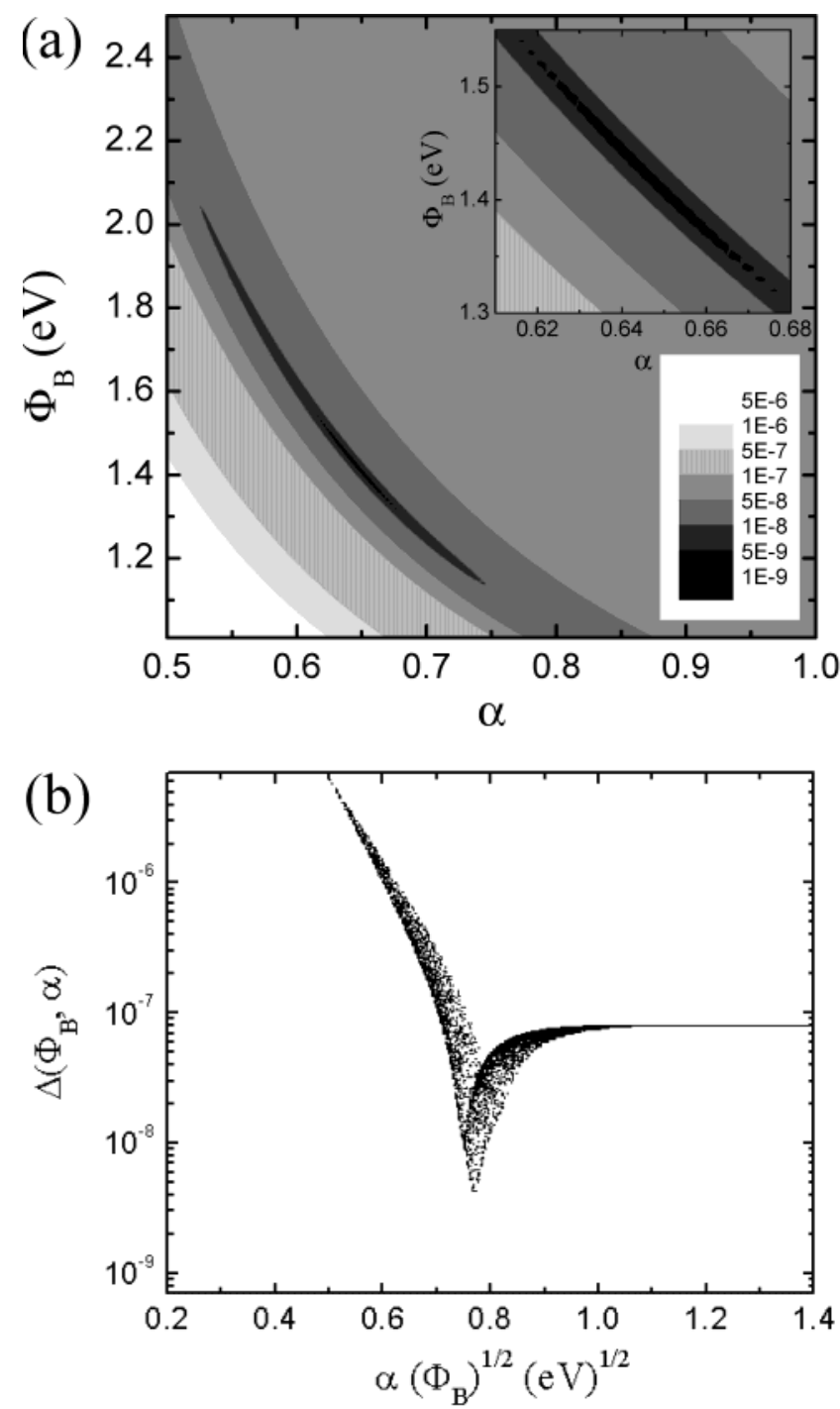

Figure 11. (a) Contour plot of $\Delta\left(\Phi_{\mathrm{B}}, \alpha\right)$ values for the $\mathrm{C} 12$ nanopore device as a function of $\Phi_{\mathrm{B}}$ and $\alpha$, where the darker region corresponds to a better fitting. Inset shows detail minimization fitting regions. (b) A plot of $\Delta\left(\Phi_{\mathrm{B}}, \alpha\right)$ as a function of $\alpha \Phi_{\mathrm{B}}^{1 / 2}$.

hence decreasing the $\beta$ value. This is particularly pronounced for applied voltages larger than $0.5 \mathrm{~V}$. As shown previously, the calculated $\beta$ values are almost independent of bias in the low-bias range $(V<\sim 0.5 \mathrm{~V}),{ }^{22}$ which gives an average $\beta=$ $0.83 \pm 0.04 \AA^{-1}$ in the low-bias region (from 0 to $0.5 \mathrm{~V}$ ) from Figure $10 \mathrm{~b}$. The $\beta$ value $\left(0.83 \pm 0.04 \AA^{-1} \approx 1.03 \pm 0.05\right.$ per methylene) for alkanethiols reported here is comparable to previously reported values as summarized in Table 1. Combining values from both $I(V, T)$ fittings (for different lengths) and length dependence gives an average values of $\Phi_{\mathrm{B}}=1.39 \pm 0.01 \mathrm{eV}$ and $\alpha=0.65 \pm 0.01 .22$

To investigate the dependency of the Simmons model on $\Phi_{\mathrm{B}}$ and $\alpha$, a fitting minimization analysis was undertaken on the individual $\Phi_{\mathrm{B}}$ and $\alpha$ values as well as their product form of $\alpha$ $\Phi_{\mathrm{B}}{ }^{1 / 2}$ in eq $2 \mathrm{~b} . \Delta\left(\Phi_{\mathrm{B}}, \alpha\right)=\left(\Sigma\left|I_{\text {exp }, \mathrm{V}}-I_{\text {cal, } \mathrm{V}}\right|^{2}\right)^{1 / 2}$ was calculated and plotted where $I_{\text {exp,V }}$ is the experimental current - voltage values and $I_{\mathrm{cal}, \mathrm{V}}$ is calculated using eq 1 . Different $\left\{\Phi_{\mathrm{B}}, \alpha\right\}$ pairs (7 500) were used in the fittings, with $\Phi_{B}$ ranging from 1.0 to $2.5 \mathrm{eV}(0.01-\mathrm{eV}$ increment $)$ and $\alpha$ from 0.5 to $1.0(0.01$ increment). Figure 11a is a representative contour plot of $\Delta\left(\Phi_{\mathrm{B}}, \alpha\right)$ vs $\Phi_{\mathrm{B}}$ and $\alpha$ values generated for the $\mathrm{C} 12 I(V)$ data where darker regions correspond to smaller $\Delta\left(\Phi_{\mathrm{B}}, \alpha\right)$ and various shades represent half order of magnitude $\Delta\left(\Phi_{\mathrm{B}}, \alpha\right)$ steps. The 
TABLE 2: Summary of Alkanethiol Tunneling Parameters in This Study

\begin{tabular}{|c|c|c|c|c|c|c|c|}
\hline platform & $\beta\left(\AA^{-1}\right)^{a}$ & alkanethiol & $J$ at $1 \mathrm{~V}(\mathrm{~A} / \mathrm{cm} 2)$ & $\Phi_{\mathrm{B}}(\mathrm{eV})$ & $\alpha$ & $m^{*}(\mathrm{~m})$ & $\beta\left(\AA^{-1}\right)^{b}$ \\
\hline \multirow[t]{3}{*}{ nanopore } & $0.83 \pm 0.04$ & $\mathrm{C} 8$ & $31000 \pm 10000$ & $1.83 \pm 0.10$ & $0.61 \pm 0.01$ & 0.37 & $0.85 \pm 0.04$ \\
\hline & & $\mathrm{C} 12$ & $1500 \pm 200$ & $1.42 \pm 0.04$ & $0.65 \pm 0.01$ & 0.42 & $0.79 \pm 0.02$ \\
\hline & & $\mathrm{C} 16$ & $23 \pm 2$ & $1.40 \pm 0.03$ & $0.68 \pm 0.01$ & 0.46 & $0.82 \pm 0.02$ \\
\hline monolithic & & C8 & $115000 \pm 14000^{c}$ & & & & \\
\hline \multirow[t]{3}{*}{ mesa } & & $\mathrm{C} 10$ & $4700 \pm 600$ & & & & \\
\hline & & $\mathrm{C} 12$ & $3300 \pm 400$ & & & & \\
\hline & & C16 & $6.6 \pm 0.8$ & & & & \\
\hline CAFM-planar & & $\mathrm{C} 12$ & $40-510^{d}$ & & & & \\
\hline
\end{tabular}

${ }^{a} \beta$ values were determined from the length-dependent tunneling analysis using eq $3 .{ }^{b} \beta$ values were calculated using eq $2 \mathrm{~b} .{ }^{c} J$ at $0.5 \mathrm{~V} .{ }^{d}$ Range of estimate $J$ (using contact junction area estimated from eq 4) for applied loading force from 5 to $20 \mathrm{nN}$.

dark regions represent better fits of eq 1 to the measured $I(V)$ data. In the inset in Figure 11a, one can see there is a range of possible $\Phi_{\mathrm{B}}$ and $\alpha$ values yielding minimum-fitting parameters. Although the tunneling parameters determined from the previous Simmons tunneling fitting $\left\{\Phi_{\mathrm{B}}=1.42 \mathrm{eV}\right.$ and $\left.\alpha=0.65\right\}$ lie within this minimum region in this figure, there is a distribution of other possible values.

A plot of $\Delta\left(\Phi_{\mathrm{B}}, \alpha\right)$ vs $\alpha \Phi_{\mathrm{B}}{ }^{1 / 2}$ for the same device reveals a more pronounced dependence and is shown in Figure 11b. This plot indicates that the fitting to the Simmons model sharply depends on the product of $\alpha \Phi_{\mathrm{B}}{ }^{1 / 2}$. For this plot, the $\Delta\left(\Phi_{\mathrm{B}}, \alpha\right)$ is minimized at $\alpha \Phi_{\mathrm{B}}{ }^{1 / 2}$ of $0.77(\mathrm{eV})^{1 / 2}$, corresponding to a $\beta$ value of $0.79 \AA^{-1}$ from eq $2 \mathrm{~b}$. This $\beta$ value agrees with the value $\left(0.83 \pm 0.04 \AA^{-1}\right)$ determined from the length-dependent analysis. The C8 and C16 nanopore devices showed similar results, indicating the Simmons tunneling model has a strong $\alpha \Phi_{\mathrm{B}}^{1 / 2}$ dependence.

\section{Comparison and Discussion}

Comparative results of transport parameters calculated from $I(V)$ measurements for various molecules from all three methods are summarized in Table 2. Current densities are presented across all three methods. $\beta$ values determined from lengthdependent tunneling analysis are presented for the nanopore method only.

The limitations of various methods for characterizing alkanethiol $\mathrm{M}-\mathrm{I}-\mathrm{M}$ junctions are apparent when comparing results. The CAFM-planar method presents a simple surface for characterization, and the use of an AFM probe allows one to potentially characterize defect-free regions of the SAM. However, it is easy to perform an "incorrect" measurement, i.e., the probe-loading force causing penetration or deformation of the molecular layer, and the contact area is not well known. Furthermore, the role of adsorbates (such as water) in the molecule-tip junction has not been quantified and makes this method less controllable and prone to systematic errors.

The monolithic mesa method has the potential to provide a large number of devices per chip. In addition, devices may be designed to be probed using automated probing methods (see Figure 2a). Fabrication methods allow large numbers of chips to be readily manufactured. Devices may be packaged and characterized using standard solid-state or IC device methods. However a wide dispersion of current density is observed in our study, indicating that other conduction mechanisms are possible, and suggests that a temperature-dependent analysis is needed. We were unable to perform this because devices could not be thermally cycled. Additionally this method was also observed to have a much lower device yield than the nanopore platform (roughly an order of magnitude lower yield $\sim 0.5 \%$ vs $\sim 5 \%$ ).

The nanopore method has been successfully used for characterizing and demonstrating molecular electronic behavior, including $I(V, T)$ characterization. ${ }^{22,27,28}$ This platform provides a pristine nanoscale electrode surface for SAM formation, which minimizes defect-dependent charge-transport effects in characterized devices. Alkanethiol SAMs formed only in the nanopore structure showed the correct bias dependence of $\beta$ values and temperature-independent $I(V)$ characteristics. Therefore, in our study, the nanopore method was observed to be the only satisfactory characterization method for molecular-scale $\mathrm{M}-\mathrm{I}-\mathrm{M}$ charge transport in alkanethiol SAMs. Because of it's ability to perform kinetic studies, this method has been recently applied to perform more detailed transport characterization techniques such as inelastic electron tunneling spectroscopic studies to investigate the coupling interactions between tunneling charge carriers with molecular vibrational modes. ${ }^{46}$

\section{Conclusions}

Charge transport through molecular $\mathrm{M}-\mathrm{I}-\mathrm{M}$ systems was investigated using three different methods: CAFM-planar, monolithic mesa, and nanopore. Molecular insulator thickness was varied in all three cases by using different-length alkanethiol SAMs. Transport parameters were determined from $I(V)$ fitting and length-dependent analysis when possible and compared to tunneling models. The nanopore method was found to be the only satisfactory method for electronic transport characterization of molecular monolayers, primarily because of this method's capability to perform variable temperature characterization.

Acknowledgment. The authors would like to thank Ilona Kretzschmar, Ryan Munden, and Azucena A. Munden for helpful discussions and assistance. This work was supported by DARPA/ONR (N00014-01-1-0657), ARO (DAAD19-01-10592), AFOSR (F49620-01-1-0358), NSF (DMR-0095215), and NASA (NCC 2-1363).

\section{References and Notes}

(1) Molecular Nanoelectronics; Reed, M. A., Lee, T., Eds.; American Scientific Publishers: Stevenson Ranch, 2003.

(2) Heath, J. R.; Ratner, M. A. Phys. Today 2003, May 43.

(3) Nitzan, A.; Ratner, M. A. Science 2003, 300, 1384.

(4) Ulman, A. An Introduction to Ultrathin Organic Films from Langmuir-Blodgett to Self-Assembly; Academic Press: Boston, 1991.

(5) Bumm, L. A.; Arnold, J. J.; Dunbar, T. D.; Allara, D. L.; Weiss, P. S. J. Phys. Chem. B 1999, 103, 8122-8127.

(6) Xu, B.; Tao, N. J. Science 2003, 301, 1221-1223. 5556.

(7) Wold, D. J.; Frisbie, C. D. J. Am. Chem. Soc. 2001, 123, 5549-

(8) Wold, D. J.; Haag, R.; Rampi, M. A.; Frisbie, C. D. J. Phys. Chem. B 2002, 106, 2813-2816.

(9) Cui, X. D.; Zarate, X.; Tomfohr, J.; Sankey, O. F.; Primak, A.; Moore, A. L.; Moore, T. A.; Gust, D.; Harris, G.; Lindsay, S. M. Nanotechnology 2002, 13, 5-14.

(10) Cui, X. D.; Primak, A.; Zarate, X.; Tomfohr, J.; Sankey, O. F.; Moore, A. L.; Moore, T. A.; Gust, D.; Nagahara, L. A.; Lindsay, S. M. J. Phys. Chem. B 2002, 106, 8609-8614. 
(11) Holmlin, R.; Haag, R.; Chabinyc, M. L.; Ismagilov, R. F.; Cohen, A. E.; Terfort, A. Rampi, M. A.; Whitesides, G. M. J. Am. Chem. Soc. 2001, 123, 5075-5085

(12) Rampi, M. A.; Whitesides, G. M. Chem. Phys. 2002, 281, 373-391.

(13) Slowinski, K.; Fong, H. K. Y.; Majda, M. J. Am. Chem. Soc. 1999, 121, 7257-7261.

(14) York, R. L.; Nguyen, P. T.; Slowinski, K. J. Am. Chem. Soc. 2003, $125,5948-5953$.

(15) Kushmerick, J. G.; Holt, D. B.; Pollack, S. K.; Ratner, M. A.; Yang, J. C.; Schull, T. L.; Naciri, J.; Moore, M. H.; Shashidhar, R. J. Am. Chem. Soc. 2002, 124, 10654-10655.

(16) Smalley, J. F.; Feldberg, S. W.; Chidsey, C. E. D.; Linford, M. R.; Newton, M. D.; Liu, Y. J. Phys. Chem. 1995, 99, 13141-13149.

(17) Weber, K.; Hockett, L.; Creager, S. J. Phys. Chem. B 1997, 101, 8286-8291.

(18) Slowinski, K.; Chamberlain, R. V.; Miller, C. J.; Majda, M. J. Am. Chem. Soc. 1997, 119, 11910-11919.

(19) Ratner, M. A.; Davis, B.; Kemp, M.; Mujica, V.; Roitberg, A.; Yaliraki, S. In Molecular Electronics: Science and Technology, The Annals of the New York Academy of Sciences; Aviram, A., Ratner, M., Eds.; The New York Academy of Sciences: New York, 1998; Vol. 852.

(20) Although the HOMO-LUMO gap of alkyl chain type molecules has been reported (see ref 21), there is no experimental data on the HOMOLUMO gap for the Au/alkanethiol SAM/Au system. The commonly used HOMO-LUMO gap of alkanethiol is $8 \mathrm{eV}$.

(21) (a) Boulas, C.; Davidovits, J. V.; Rondelez, F.; Vuillaume, D. Phys. Rev. Lett. 1996, 76, 4797-4800. (b) Fujihira, M; Inokuchi, H. Chem. Phys. Lett. 1972, 17, 554-556. (c) Lias, S. G.; Bartmess, J. E.; Liebman, J. F.; Holmes, J. L.; Levin, R. D.; Mallard, W. G. Gas-Phase Ion and Neutral Thermochemistry. J. Phys. Chem. Ref. Data 1998, 17 (1), 24

(22) Wang, W.; Lee, T.; Reed, M. A. Phys. Rev. B 2003, 68, 035416.

(23) Fan, F. F.; Yang, J.; Cai, L.; Price, D. W.; Dirk, S. M.; Kosynkin, D. V.; Yao, Y.; Rawlett, A. M.; Tour, J. M.; Bard, A. J. J. Am. Chem. Soc 2002, 124, 5550-5560.

(24) Kaun, C.-C.; Guo, H. Nano Lett. 2003, 3, 1521-1525.

(25) Piccinin, S.; Selloni, A.; Scandolo, S.; Car, R.; Scoles, G. J. Chem. Phys. 2003, 119, 6729-6735.

(26) Tomfohr, J. K.; Sankey, O. F. Phys. Rev. B 2002, 65, 245105.

(27) Zhou, C.; Deshpande, M. R.; Reed, M. A.; Jones, L., II; Tour, J. M. Appl. Phys. Lett. 1997, 71, 611-613.

(28) Chen, J.; Reed, M. A.; Rawlett, A. M.; Tour, J. M. Science 1999, $286,1550-1552$.
(29) Ethanol and alkanethiols were purchased from Sigma-Aldrich.

(30) Ralls, K. S.; Buhrman, R. A.; Tiberio, R. C. Appl. Phys. Lett. 1989 $55,2459-2461$

(31) AFM system used in this study is ThermoMicroscope (CP-Research model) ambient AFM/STM system from Veeco Instruments Inc.

(32) The CAFM probe is $\mathrm{Au}(20 \mathrm{~nm}) / \mathrm{Cr}(20 \mathrm{~nm})$ coated AFM probe. Its nominal force constant is $0.35 \mathrm{~N} / \mathrm{m}$. The radius of the probe tip is $90 \pm 20$ $\mathrm{nm}$ determined from SEM images.

(33) Metzger, R. M.; Chen, B.; Höpfner, U.; Lakshmikantham, M. V.; Vuillaume, D.; Kawai, T.; Wu, X.; Tachibana, H.; Hughes, T. V.; Sakurai, H.; Baldwin, J. W.; Hosch, C.; Cava, M. P.; Brehmer, L.; Ashwell, G. J. J. Am. Chem. Soc. 1997, 119, 10455-10466.

(34) Simmons, J. G. J. Appl. Phys. 1963, 34, 1793-1803.

(35) Franz two-band model can also explain the alkanethiol tunneling characteristics. See ref 22.

(36) Joachim, C.; Magoga, M. Chem. Phys. 2002, 281, 347-352.

(37) (a) Yamamoto, H.; Waldeck, D. H. J. Phys. Chem. B 2002, 106, 7469-7473. (b) Napper, A. M.; Liu, Haiying.; Waldeck, D. H. J. Phys. Chem. B 2001, 105, 7699-7707.

(38) Johnson, K. L. Contact Mechanics; Cambridge University Press: 1985.

(39) (a) Weihs, T. P.; Nawaz, Z.; Jarvis, S. P.; Pethica, J. B. Appl. Phys. Lett. 1991, 59, 3536-3538. (b) Johnson, K. L.; Kendall, K.; Robert, A. D. Proc. R. Soc. London A. 1971, 324, 301-313.

(40) (a) Henda, R.; Grunze, M.; Pertsin, A. J. Tribology Lett. 1998, 5 , 191-195. (b) Salvadori, M. C.; Brown, I. G.; Vaz, A. R.; Melo, L. L.; Cattani, M. Phys. Rev. B 2003, 67, 153404. (c) The Poisson ratio for most materials is between 0.25 and 0.5 .

(41) Tivanski, A. V.; Bemis, J. E.; Akhremitchev, B. B.; Liu, H.; Walker, G. C. Langmuir 2003, 19, 1929-1934.

(42) Son, K. A.; Kim, H. I.; Houston, J. E. Phys. Rev. Lett. 2001, 86, 5357-5360.

(43) Wold and Frisbie (ref 7) have reported a transition of the slope from -0.83 to -7.5 when the loading force becomes larger than $70 \mathrm{nN}$ on the alkanethiol-coated Au substrate at which an abrupt change of SAM structure was assumed.

(44) The C8 device degraded after a higher bias $(>0.5 \mathrm{~V})$ was applied.

(45) Wang, W.; Lee, T.; Kamdar, M.; Reed, M. A.; Stewart, M. P.; Hwang, J. J.; Tour, J. M. Superlattices Microstruct. 2003, 33, 217-226. (46) Wang, W.; Lee, T.; Kretzschmar, I.; Reed, M. A. Nano Lett. 2004, 4, 643-646.

(47) Private communication with H. Guo at McGill University, Canada. 